\title{
On the structure of $D_{S J}^{*}(2317)$ and $D_{S J}(2460)$
}

\section{Pietro Colangelo*}

Istituto Nazionale di Fisica Nucleare, Sezione di Bari, Italy

E-mail: pietro.colangelo@ba.infn.it

\section{Fulvia De Fazio}

Istituto Nazionale di Fisica Nucleare, Sezione di Bari, Italy

E-mail: fulvia.defazio@ba.infn.it

\section{Rossella Ferrandes}

Dipartimento di Fisica dell'Universitá di Bari and INFN, Sezione di Bari, Italy

E-mail: rossella.ferrandes@ba.infn.it

\section{Altug Ozpineci}

INFN, Sezione di Bari, Italy and METU University, Ankara, Turkey

E-mail: ozpineci@ba.infn.it

We discuss the structure of the narrow mesons with charm and strangeness $D_{S J}^{*}(2317)$ and $D_{S J}(2460)$, stressing the role of the radiative decay modes in shedding light on the quark content of these states. We argue that present experimental data favour the interpretation of the two states as ordinary $\bar{c} s$ mesons. We suggest the existence of resonances with similar properties and well predicted masses in the $\bar{b} s$ sector.

International Europhysics Conference on High Energy Physics

July 21st - 27th 2005

Lisboa, Portugal

\footnotetext{
* Speaker.
} 


\section{Introduction}

The observation of two narrow resonances with charm and strangeness, $D_{s J}^{*}(2317)$ in the $D_{s} \pi^{0}$ channel and $D_{S J}(2460)$ in the $D_{s}^{*} \pi^{0}$ and $D_{s} \gamma$ channels [1], has raised discussions about the quark structure of these states [2]. Their identification with the scalar and axial vector $\bar{c} s$ mesons $\left(D_{s 0}\right.$ and $\left.D_{s 1}^{\prime}\right)$ is natural; in the $m_{c} \rightarrow \infty$ limit such states are expected to be degenerate in mass and to form a $s_{\ell}^{P}=\frac{1}{2}^{+}$doublet, with $s_{\ell}$ the angular momentum of the light degrees of freedom. In that interpretation $D_{S J}^{*}(2317), D_{s J}(2460), D_{s 1}(2536)$ and $D_{s 2}(2573)$ are the four lowest lying Pwave $\bar{c} s$ states. However, quark model estimates of the masses of these mesons generally produce larger values than the measured ones, implying that the two $\bar{c} s$ scalar and axial-vector $D_{s 0}$ and $D_{s 1}^{\prime}$ states should be heavy enough to decay to $D K$ and $D^{*} K$. Moreover, the $B \rightarrow D_{S J}^{*}(2317) D$ and $B \rightarrow D_{s J}(2460) D$ decay rates computed by naive factorization do not agree with experiment [3]. On this basis, other interpretations for $D_{S J}^{*}(2317)$ and $D_{s J}(2460)$ have been proposed, namely that of molecular states [4]. Radiative transitions probe the structure of hadrons and are suitable to understand the nature of $D_{S J}^{*}(2317)$ and $D_{S J}(2460)$ since the rates can be predicted by various methods and compared to the experimental findings $[5,6]$. One method is Light-Cone QCD sum rules [7].

\section{Radiative decays by Light-Cone QCD Sum Rules}

The $D_{s J}^{*}(2317) \rightarrow D_{s}^{*} \gamma$ and $D_{s J}(2460) \rightarrow D_{s}^{(*)} \gamma, D_{s J}^{*}(2317) \gamma$ decay amplitudes:

$$
\begin{aligned}
\left\langle\gamma(q, \lambda) D_{s}^{*}\left(p, \lambda^{\prime}\right) \mid D_{s 0}(p+q)\right\rangle & =e d\left[\left(\varepsilon^{*} \cdot \tilde{\eta}^{*}\right)(p \cdot q)-\left(\varepsilon^{*} \cdot p\right)\left(\tilde{\eta}^{*} \cdot q\right)\right] \\
\left\langle\gamma(q, \lambda) D_{s}(p) \mid D_{s 1}^{\prime}\left(p+q, \lambda^{\prime \prime}\right)\right\rangle & =e g_{1}\left[\left(\varepsilon^{*} \cdot \eta\right)(p \cdot q)-\left(\varepsilon^{*} \cdot p\right)(\eta \cdot q)\right] \\
\left\langle\gamma(q, \lambda) D_{s}^{*}\left(p, \lambda^{\prime}\right) \mid D_{s 1}^{\prime}\left(p+q, \lambda^{\prime \prime}\right)\right\rangle & =i e g_{2} \varepsilon_{\alpha \beta \sigma \tau} \eta^{\alpha} \tilde{\eta}^{* \beta} \varepsilon^{* \sigma} q^{\tau} \\
\left\langle\gamma(q, \lambda) D_{s 0}(p) \mid D_{s 1}^{\prime}\left(p+q, \lambda^{\prime \prime}\right)\right\rangle & =i e g_{3} \varepsilon_{\alpha \beta \sigma \tau} \varepsilon^{* \alpha} \eta^{\beta} p^{\sigma} q^{\tau}
\end{aligned}
$$

$\left(\varepsilon(\lambda)\right.$ is the photon polarization vector and $\tilde{\eta}\left(\lambda^{\prime}\right), \eta\left(\lambda^{\prime \prime}\right)$ the $D_{s}^{*}$ and $D_{s 1}^{\prime}$ polarization vectors) involve the hadronic parameters $d, g_{1}, g_{2}$ and $g_{3}$. They can be determined by Light-Cone Sum rules $[9,10]$, starting from the light-cone expansion of correlation functions

$$
F(p, q)=i \int d^{4} x e^{i p \cdot x}\left\langle\gamma(q, \lambda)\left|T\left[J_{A}^{\dagger}(x) J_{B}(0)\right]\right| 0\right\rangle
$$

of quark-antiquark currents $J_{A, B}$ having the same quantum number of the decaying and of the produced charmed mesons, and an external photon state of momentum $q$ and helicity $\lambda$. In this expansion the perturbative photon coupling to the strange and charm quarks is considered together with the contributions of the photon emission from the soft $s$ quark, expressed as photon matrix elements of increasing twist, fig.1 [11]. The correlation function can also be expressed in terms of the contribution of the lowest-lying resonances, the current-vacuum matrix elements of which are computed by the same method [12], and a continuum of states which is treated invoking global quark-hadron duality. A Borel transformation introduces an external parameter $M^{2}$, the hadronic quantities being independent of it. The results depicted in fig. 2 correspond to the rates in Table 1.

The main result is that the rate of $D_{s J}(2460) \rightarrow D_{s} \gamma$ is the largest one among the radiative $D_{S J}(2460)$ rates, and this is confirmed by experiment, see Table 2 . Since this result comes from 


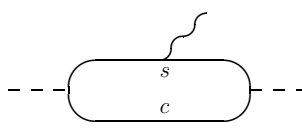

(a)

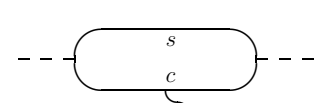

(b)

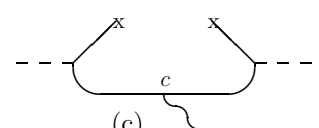

(c)

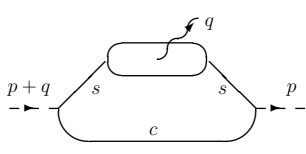

(a)

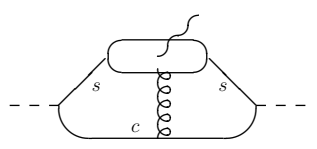

(b)

Figure 1: Diagrams describing the perturbative photon emission by the strange and charm quark ( (a,b) in the first line) and two and three-particle photon distribution amplitudes (second line); (c) corresponds to the strange quark condensate contribution.
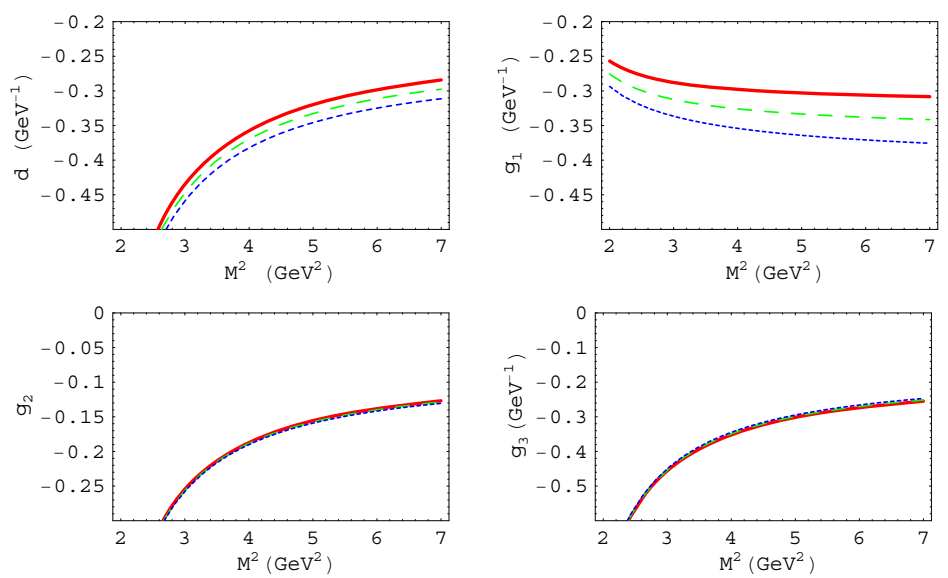

Figure 2: Sum rule results for the hadronic parameters in eqs.(2.1)-(2.4). $M^{2}$ is the Borel parameter.

a description of the mesons as quark-antiquark states, we consider it as a quantitative argument in support of a $\bar{c} s$ interpretation of $D_{s J}^{*}(2317)$ and $D_{s J}(2460)$. The observation of all the radiative decay modes with the predicted relative rates would reinforce the conclusion. In order to quantitatively understand the experimental data in Table 2 one should precisely know the widths of the isospin violating transitions $D_{s 0} \rightarrow D_{s} \pi^{0}$ and $D_{s 1}^{\prime} \rightarrow D_{s}^{*} \pi^{0}$. In the description of these transitions based on the mechanism of $\eta-\pi^{0}$ mixing $[13,4,5,6]$ one should accurately determine the strong couplings $D_{s 0} D_{s} \eta$ and $D_{s 0}^{\prime} D_{s}^{*} \eta$ for finite heavy quark mass and including $S U(3)$ corrections.

\section{Narrow states in the beauty sector}

The mass estrapolation from charm to beauty and the interpretation of $D_{S J}^{*}(2317)$ and $D_{s J}(2460)$ as ordinary mesons allow to predict the masses of corresponding states in the beauty sector: $M\left(B_{s 0}\right)=$ $5.71 \pm 0.03 \mathrm{GeV}$ and $M\left(B_{s 1}^{\prime}\right)=5.77 \pm 0.03 \mathrm{GeV}$ [14]. Therefore, $B_{s 0}$ and $B_{s 1}^{\prime}$ should be respectively below the $B K$ and $B^{*} K$ thresholds; they should be observed as narrow peaks in $B_{s} \pi^{0}$ and $B_{s}^{*} \pi^{0}, B_{s}^{(*)} \gamma$ invariant mass distributions, a possible observation at the Tevatron and the LHC. 
Table 1: Radiative decay widths (in $\mathrm{keV}$ ) of $D_{S J}^{*}(2317)$ and $D_{S J}(2460)$ obtained by Light-Cone sum rules (LCSR). Vector Meson Dominance (VMD) and constituent quark model (QM) results are also reported.

\begin{tabular}{cccccc}
\hline Initial state & Final state & LCSR [7] & VMD [2, 6] & QM [5] & QM [8] \\
\hline$D_{s J}^{*}(2317)$ & $D_{s}^{*} \gamma$ & $4-6$ & 0.85 & 1.9 & 1.74 \\
$D_{s J}(2460)$ & $D_{s} \gamma$ & $19-29$ & 3.3 & 6.2 & 5.08 \\
& $D_{s}^{*} \gamma$ & $0.6-1.1$ & 1.5 & 5.5 & 4.66 \\
& $D_{S J}^{*}(2317) \gamma$ & $0.5-0.8$ & - & 0.012 & 2.74 \\
\hline
\end{tabular}

Table 2: Measurements and 90\% CL upper limits of ratios of $D_{S J}^{*}(2317)$ and $D_{S J}(2460)$ decay widths.

\begin{tabular}{cccc}
\hline & Belle & BaBar & CLEO \\
\hline$\Gamma\left(D_{s J}^{*}(2317) \rightarrow D_{s}^{*} \gamma\right) / \Gamma\left(D_{s J}^{*}(2317) \rightarrow D_{s} \pi^{0}\right)$ & $<0.18$ & - & $<0.059$ \\
$\Gamma\left(D_{s J}(2460) \rightarrow D_{s} \gamma\right) / \Gamma\left(D_{s J}(2460) \rightarrow D_{s}^{*} \pi^{0}\right)$ & $0.45 \pm 0.09$ & $0.30 \pm 0.04$ & $<0.49$ \\
$\Gamma\left(D_{s J}(2460) \rightarrow D_{s}^{*} \gamma\right) / \Gamma\left(D_{s J}(2460) \rightarrow D_{s}^{*} \pi^{0}\right)$ & $<0.31$ & - & $<0.16$ \\
$\Gamma\left(D_{s J}(2460) \rightarrow D_{s J}^{*}(2317) \gamma\right) / \Gamma\left(D_{s J}(2460) \rightarrow D_{s}^{*} \pi^{0}\right)$ & - & $<0.23$ & $<0.58$ \\
\hline
\end{tabular}

\section{References}

[1] B. Aubert et al. [BABAR], Phys. Rev. Lett. 90 (2003) 242001. D. Besson et al. [CLEO], Phys. Rev. D68 (2003) 032002. Y. Mikami et al. [Belle], Phys. Rev. Lett. 92 (2004) 012002. P. Krokovny et al. [Belle], Phys. Rev. Lett. 91 (2003) 262002. A. Drutskoy et al. [Belle], Phys. Rev. Lett. 96 (2005) 061802. E. W. Vaandering [FOCUS], hep-ex/0406044. B. Aubert et al. [BABAR], Phys. Rev. Lett. 93 (2004) 181801. B. Aubert et al. [BABAR], Phys. Rev. D69 (2004) 031101. B. Aubert et al. [BABAR], hep-ex/0408067.

[2] For a review see: P. Colangelo, F. De Fazio and R. Ferrandes, Mod. Phys. Lett. A 19 (2004) 2083.

[3] A. Datta and P. J. O’donnell, Phys. Lett. B 572 (2003) 164.

[4] T. Barnes, F. E. Close and H. J. Lipkin, Phys. Rev. D 68 (2003) 054006.

[5] S. Godfrey, Phys. Lett. B 568 (2003) 254.

[6] P. Colangelo and F. De Fazio, Phys. Lett. B 570 (2003) 180.

[7] P. Colangelo, F. De Fazio and A. Ozpineci, Phys. Rev. D 72 (2005) 074004.

[8] W. A. Bardeen, E. J. Eichten and C. T. Hill, Phys. Rev. D 68 (2003) 054024.

[9] V. M. Belyaev et al., Phys. Rev. D 51 (1995) 6177, and references therein.

[10] For a review see: P. Colangelo and A. Khodjamirian, arXiv:hep-ph/0010175.

[11] P. Ball, V. M. Braun and N. Kivel, Nucl. Phys. B 649 (2003) 263.

[12] P. Colangelo et al., Phys. Rev. D 52 (1995) 6422. P. Colangelo and F. De Fazio, Eur. Phys. J. C 4 (1998) 503. P. Colangelo, F. De Fazio and N. Paver, Phys. Rev. D 58 (1998) 116005.

[13] P. L. Cho and M. B. Wise, Phys. Rev. D 49 (1994) 6228.

[14] P. Colangelo, F. De Fazio and R. Ferrandes, BARI-TH/526-05. 